\title{
CONVEXITY PROPERTIES OF SOLUTIONS TO THE FREE SCHRÖDINGER EQUATION WITH GAUSSIAN DECAY
}

\author{
L. Escauriaza, C.E. Kenig, G. Ponce, and L. Vega \\ AbStRaCt. We study convexity properties of solutions to the free Schrödinger equation \\ with Gaussian decay.
}

\section{Introduction}

The main purpose of this note is to study convexity properties of two classes of functions. The first one is the class of functions which, together with their Fourier transform, have Gaussian decay. The second class is the class of functions for which the free Schrödinger evolution has Gaussian decay at two different times. We will see later that the two classes coincide (see Theorem 1 below). Moreover, (vii) in Theorem 1 show that this is a large class of functions. As motivation for the study of these issues, we recall the well known "uncertainty principle" due to G. H. Hardy (see $[10])$ :

(A) Assume $n=1, f(x)=O\left(e^{-A x^{2}}\right)$ and $\hat{f}(\xi)=O\left(e^{-B \xi^{2}}\right)$. If $A B>1 / 4$, then $f \equiv 0$. Moreover, if $A B=1 / 4$, then $f(x)=c e^{-A x^{2}}$, for some constant $c$. In [9] this result was extended to higher dimensions, with $x^{2}$ and $\xi^{2}$ replaced by $|x|^{2}$ and $|\xi|^{2}$.

Here

$$
\widehat{f}(\xi)=(2 \pi)^{-\frac{n}{2}} \int_{\mathbb{R}^{n}} e^{-i x \cdot \xi} f(x) d x .
$$

As pointed out in [4], this result has an equivalent formulation for the free Schrödinger equation. Thus, consider the IVP for the free Schrödinger equation

$$
\left\{\begin{array}{l}
\partial_{t} u=i \Delta u, \quad x \in \mathbb{R}^{n}, t \in \mathbb{R} \\
u(x, 0)=u_{0}(x)
\end{array}\right.
$$

whose solution $u(x, t)=e^{i t \Delta} u_{0}(x)$ can be written as

$$
\begin{aligned}
& u(x, t)=\left(e^{-i|\xi|^{2} t} \widehat{u}_{0}\right)^{\vee}(x)=\int_{\mathbb{R}^{n}} \frac{e^{i|x-y|^{2} / 4 t}}{(4 \pi i t)^{n / 2}} u_{0}(y) d y \\
= & \frac{e^{i|x|^{2} / 4 t}}{(4 \pi i t)^{n / 2}} \int_{\mathbb{R}^{n}} e^{-2 i x \cdot y / 4 t} e^{i|y|^{2} / 4 t} u_{0}(y) d y=\frac{e^{i|x|^{2} / 4 t}}{(2 i t)^{n / 2}}\left(e^{\left.i \cdot \widehat{\mid}\right|^{2} / 4 t} u_{0}\right)\left(\frac{x}{2 t}\right),
\end{aligned}
$$

i.e. if $c_{t}=(2 i t)^{n / 2}$, then

$$
c_{t} e^{-i|x|^{2} / 4 t} u(x, t)=\left(e^{i|\cdot|^{2} / 4 t} u_{0}\right)^{\wedge}\left(\frac{x}{2 t}\right) .
$$

Received by the editors October 10, 2007.

The first and fourth authors are supported by MEC grant MTM2007-62186. The second and third authors were supported by NSF grants. 
Formula (1.2) tells us that $e^{-i|x|^{2} / 4 t} u(x, t)$ is a multiple of a rescaled Fourier transform of $e^{i|y|^{2} / 4 t} u_{0}(y)$, thus Hardy's result can be restated in terms of the Schrödinger equation as:

$\left(\mathrm{A}^{\prime}\right)$ If $u_{0}(x)=O\left(e^{-|x|^{2} / \beta^{2}}\right), u(x, t)=e^{i t \Delta} u_{0}(x)=O\left(e^{-|x|^{2} / \alpha^{2}}\right), t>0$ and $\alpha \beta<$ $4 t$, then $u \equiv 0$ in $\mathbb{R}^{n} \times[0, t]$. Moreover, if $\alpha \beta=4 t$, then $u$ is the solution with initial data, $\omega e^{-\left(\frac{1}{\beta^{2}}+\frac{i}{4 t}\right)|x|^{2}}$, for some complex number $\omega$.

We also recall the following extension of (A) established in [9]:

(B) If $e^{A_{1}|\cdot|^{2}} f \in L^{p}(\mathbb{R})$ and $e^{A_{2}|\cdot|^{2}} \widehat{f} \in L^{q}(\mathbb{R}), p, q \in[1, \infty]$, with at least one of them finite and with $A_{1} A_{2} \geq 1 / 4$, then $f \equiv 0$,

and the Beurling-Hörmander result in [6]:

(C) If $f \in L^{1}(\mathbb{R})$ and $\int_{\mathbb{R}} \int_{\mathbb{R}}|f(x)||\widehat{f}(\xi)| e^{|x \xi|} d x d \xi<\infty$, then $f \equiv 0$ (for extensions of this result to higher dimensions see [1]).

Because of (1.2), (B) and (C) can be rephrased as:

(B') If $u_{0} \in L^{p}\left(e^{p x^{2} / \beta^{2}} d x\right)$ and $e^{i t \partial_{x}^{2}} u_{0} \in L^{q}\left(e^{q x^{2} / \alpha^{2}} d x\right), p, q \in[1, \infty]$, with at least one of them finite and $4 t \geq \alpha \beta$, then $u_{0} \equiv 0$,

and

$\left(\mathrm{C}^{\prime}\right)$ If $u_{0} \in L^{1}(\mathbb{R})$ and $\int_{\mathbb{R}} \int_{\mathbb{R}}\left|u_{0}(x) \| e^{i t \partial_{x}^{2}} u_{0}(y)\right| e^{|x y| / 2 t} d x d y<\infty$, then $u_{0} \equiv 0$.

In [4], we obtained the following results which can be seen as variants of Hardy's uncertainty principle, in the context of Schrödinger equations with potential and for non-linear Schrödinger equations.

(I) Let $u \in C\left([0,1]: H^{2}\left(\mathbb{R}^{n}\right)\right)$ be a strong solution of

$$
i \partial_{t} u+\Delta u=V u
$$

with

$$
V: \mathbb{R}^{n} \times[0,1] \rightarrow \mathbb{C}, \quad V \in L^{\infty}\left(\mathbb{R}_{x}^{n} \times[0,1]\right), \quad \nabla_{x} V \in L_{t}^{1}\left([0,1]: L^{\infty}\left(\mathbb{R}^{n}\right)\right),
$$

and

$$
\lim _{r \uparrow \infty}\|V\|_{L_{t}^{1} L_{x}^{\infty}\{|x|>r\}}=\lim _{r \uparrow \infty} \int_{0}^{1} \sup _{|x|>r}|V(x, t)| d t=0 .
$$

There exists $c_{0}=c_{0}\left(n ;\|u\|_{L_{t}^{\infty} H_{x}^{2}} ;\|V\|_{L_{t, x}^{\infty}} ;\left\|\nabla_{x} V\right\|_{L_{t}^{1} L_{x}^{\infty}}\right)>0$ such that if

$$
u_{0}=u(0), u_{1}=u(1) \in H^{1}\left(e^{a|x|^{2}} d x\right),
$$

with $a \geq c_{0}$, then $u \equiv 0$.

(II) Let $u_{1}, u_{2} \in C\left([0,1]: H^{k}\left(\mathbb{R}^{n}\right)\right), k>n / 2+1$ be solutions of

$$
i \partial_{t} u+\Delta u+F(u, \bar{u})=0
$$

with

$$
F: \mathbb{C}^{2} \rightarrow \mathbb{C}, \quad F \in C^{k}, \quad F(0)=\partial_{u} F(0)=\partial_{\bar{u}} F(0)=0 .
$$

There exists $c_{0}=c_{0}\left(n ;\left\|u_{1}\right\|_{L_{t}^{\infty} H_{x}^{2}} ;\left\|u_{2}\right\|_{L_{t}^{\infty} H_{x}^{2}} ;\|F\|_{C^{k}}\right)>0$ such that if

$$
u_{1}(0)-u_{2}(0), \quad u_{1}(1)-u_{2}(1) \in H^{1}\left(e^{a|x|^{2}} d x\right),
$$

with $a \geq c_{0}$, then $u_{1} \equiv u_{2}$. 
In (1.4)-(1.6) we used the notation $f \in H^{1}\left(e^{a|x|^{2}} d x\right)$ if $f, \partial_{x_{j}} f \in L^{2}\left(e^{a|x|^{2}} d x\right)$ for $j=1, \ldots, n$, i.e.

$$
\int_{\mathbb{R}^{n}}|f(x)|^{2} e^{a|x|^{2}} d x+\sum_{j=1}^{n} \int_{\mathbb{R}^{n}}\left|\partial_{x_{j}} f(x)\right|^{2} e^{a|x|^{2}} d x<\infty .
$$

By fixing $u_{2} \equiv 0$ the above question relates to the persistence properties of solutions of (1.3) and (1.5), i.e. if $u(x, 0)=u_{0}(x) \in X$ (function space), then the solution $u=u(x, t)$ of $(1.3)$ (resp. (1.5)) satisfies that

$$
u \in C([0, T]: X) .
$$

These persistence properties, as part of the standard notion of well-posedness, have been studied in function spaces $X$ describing the regularity and decay of their elements. For example, they have been established in classical Sobolev spaces $X=$ $H^{s}\left(\mathbb{R}^{n}\right), s \geq s_{0}$, with $s_{0}$ the optimal Sobolev exponent, which depends on $V$ (resp. $F$ ), $n$, and $T$, with $T<\infty$, corresponding to local solutions and $T=\infty$ corresponding to global ones, and in their weighted versions, $X=H^{s}\left(\mathbb{R}^{n}\right) \cap L^{2}\left(|x|^{m} d x\right)$, with $V \in C^{[s+1]}$ (resp. $F \in C^{[s+1]}$ ), where persistence holds if $s \geq s_{0}$ and $s \geq m$, due to the fact that $\Gamma_{j}=x_{j}-2 i t \partial_{x_{j}}, j=1, . ., n$, commutes with $\partial_{t}-i \Delta$, (for details see [5] and references therein). In the case, $X=H^{s}\left(\mathbb{R}^{n}\right) \cap L^{2}\left(|x|^{m} d x\right)$, when $m>s$, persistence fails even in the free case, i.e. $V \equiv 0$ in $(1.3)$, and the extra-decay " $m-s$ " is transformed into "local regularity".

Combining the above remarks and the formula (1.2) one has that

if $u_{0} \in C_{0}^{\infty}\left(\mathbb{R}^{n}\right)$, then for any $t \neq 0, \epsilon>0 \quad e^{i t \Delta} u_{0} \in \mathbb{S}\left(\mathbb{R}^{n}\right)-L^{1}\left(e^{\epsilon|x|} d x\right)$.

In this case $u_{0} \in C_{0}^{\infty}\left(\mathbb{R}^{n}\right)$, for $t \neq 0 u(x, t)$ has an analytic extension to $\mathbb{C}^{n}$, so roughly speaking, one can say that the decay, which does not persist with the solution, is transformed into "local regularity".

One of the key results in [4] established that solutions of Schrödinger equations with potential having $L^{2}$-Gaussian decay at two different times $t_{1}$, $t_{2}$, with $t_{1}<t_{2}$, preserve this property in the time interval $\left[t_{1}, t_{2}\right]$ with a fixed Gaussian weight (see Corollary 2.2 in [4] and comments after it). In this paper, we examine in detail the case of the free particle. We shall try to understand the possible persistence properties of the solutions of the free Schrödinger equation in function spaces with exponential decay at infinity.

We can summarize part of the results in this work in the following qualitative terms:

Theorem 1. For $u_{0} \in \mathbb{S}^{\prime}\left(\mathbb{R}^{n}\right)$ the following seven statements are equivalent:

(i) There are two different real numbers $t_{1}$ and $t_{2}$, such that $e^{i t_{j} \Delta} u_{0} \in$ $L^{2}\left(e^{a_{j}|x|^{2}} d x\right)$, for some $a_{j}>0, j=1,2$.

(ii) $u_{0} \in L^{2}\left(e^{b_{1}|x|^{2}} d x\right)$ and $\widehat{u}_{0} \in L^{2}\left(e^{b_{2}|x|^{2}} d x\right)$, for some $b_{j}>0, j=1,2$.

(iii) There is $\nu:[0,+\infty) \longrightarrow(0,+\infty)$, such that $e^{i t \Delta} u_{0} \in L^{2}\left(e^{\nu(t)|x|^{2}} d x\right)$, for all $t \geq 0$.

(iv) $g(x) \equiv e^{i \tau|x|^{2}} u_{0}(x), \tau \in \mathbb{R}$, verifies (ii) with possibly different constants $b_{1}, b_{2}>0$.

(v) $u_{0}(x+i y)$ is an entire function such that $\left|u_{0}(x+i y)\right| \leq N e^{-a|x|^{2}+b|y|^{2}}$ for some constants $N, a, b>0$.

(vi) $\widehat{u}_{0}(\xi+i \eta)$ verifies $(v)$ with possibly different constants $N, a, b>0$. 
(vii) there exist $\delta, \epsilon>0$ and $h \in L^{2}\left(e^{\epsilon|x|^{2}} d x\right)$ such that $u_{0}(x)=e^{\delta \Delta} h$.

Notice that Theorem 1 characterizes the functions with Gaussian decay whose Fourier transform also has Gaussian decay and part (vii) affirms that this is a large class of functions. Our proof of (ii) implies (iii) in Theorem 1 will be a consequence of the new quantitative results in this work: some logarithmically convex inequalities for exponentially weighted $L^{2}$-norms of solutions of the free Schrödinger equation. In particular, it will be a consequence of the following Theorem:

Theorem 2. Let $\alpha$ and $\beta$ be two positive numbers and $u$ be the solution of the initial value problem

$$
\left\{\begin{array}{l}
\partial_{t} u=i \Delta u, \quad t>0, \quad x \in \mathbb{R}^{n}, \\
u(x, 0)=u_{0}(x) .
\end{array}\right.
$$

Let $T>0$. If either the right hand side of (1.9) or (1.10) are finite, then the following inequalities hold

$$
\begin{gathered}
\left\|e^{\frac{\lambda \cdot x}{\alpha t+\beta}} u(t)\right\|_{2} \leq\left\|e^{\frac{\lambda \cdot x}{\beta}} u(0)\right\|_{2}^{\theta(t)}\left\|e^{\frac{\lambda \cdot x}{\alpha T+\beta}} u(T)\right\|_{2}^{1-\theta(t)}, \quad 0 \leq t \leq T, \\
\left\|e^{\frac{\lambda \cdot x}{\alpha t+\beta}} u(t)\right\|_{2} \leq\left\|e^{\frac{\lambda \cdot x}{\beta}} u(0)\right\|_{2}^{\mu(t)}\left\|e^{\frac{2 \lambda \cdot \xi}{\alpha}} \widehat{u}(0)\right\|_{2}^{1-\mu(t)},
\end{gathered}
$$

for any $\lambda \in \mathbb{R}^{n}$, and

$$
\begin{gathered}
\left\|e^{\frac{|x|^{2}}{(\alpha t+\beta)^{2}}} u(t)\right\|_{2} \leq\left\|e^{\frac{|x|^{2}}{\beta^{2}}} u(0)\right\|_{2}^{\theta(t)}\left\|e^{\frac{|x|^{2}}{(\alpha T+\beta)^{2}}} u(T)\right\|_{2}^{1-\theta(t)}, \quad 0 \leq t \leq T, \\
\left\|e^{\frac{|x|^{2}}{(\alpha t+\beta)^{2}}} u(t)\right\|_{2} \leq\left\|e^{\frac{|x|^{2}}{\beta^{2}}} u(0)\right\|_{2}^{\mu(t)}\left\|e^{\frac{4|\xi|^{2}}{\alpha^{2}}} \widehat{u}(0)\right\|_{2}^{1-\mu(t)},
\end{gathered}
$$

where

$$
\theta(t)=\frac{\beta(T-t)}{T(\alpha t+\beta)}, \quad \text { and } \quad \mu(t)=\frac{\beta}{\alpha t+\beta} .
$$

As a convexity statement, the conclusions in Theorem 2 are empty when the extremal values (right hand side) are not finite.

From $\left(\mathrm{A}^{\prime}\right),\left(\mathrm{B}^{\prime}\right)$ and $\left(\mathrm{C}^{\prime}\right)$, one has that the terms in (1.10) vanish if $\alpha \beta$ is small enough. The same applies to (1.9) for small values of $\alpha$ and $\beta$ depending on $T$. In particular, from $\left(\mathrm{B}^{\prime}\right)$ in the $1-d$ case, one has that if $4 T \geq \beta(\alpha T+\beta)$, then the terms in (1.9) are zero, and if $4 \geq \alpha \beta$, then the terms in (1.10) are zero.

In Lemma 2 we study the class of functions with Gaussian decay whose Fourier transform also has Gaussian decay. We show that this class is an algebra with respect to the pointwise product of functions and that it is closed under the action of the Schrödinger group. Therefore, it is also closed with respect to convolutions and multiplication by functions of the form $e^{i a|x|^{2}}, a \in \mathbb{R}$. As a consequence, in Corollary 1, we show that if a free Schrödinger solution has Gaussian decay at two different times, then the data belongs to this class, i.e. $(i)$ implies $(i i)$ in Theorem 1.

Section 2 contains the proofs of Theorems 1 and Theorem 2. The proof of Theorem 2 will be based on a general abstract result given in Theorem 3 .

Once Theorem 2 has been proved we obtain some generalizations and consequences of it. Corollaries 2-4 are extensions of the estimates (1.9)-(1.10). In Corollaries 5 and 6 we apply (1.9)-(1.10) to any pair (or any finite set) of solutions of the free Schrödinger 
equation. These estimates, which describe the interaction of two solutions, are somehow similar in spirit to those found in [2, Sections 10 and 11]. Corollary 7 is an application of the results in Theorem 1.2 and of the Galilean invariance property of the free Schrödinger equation. These extensions and applications are in section 3.

Finally, we explain and outline some extensions of the results in sections 2 and 3 to the case of a non-zero potential. These results will be studied in more detail in a forthcoming publication.

Acknowledgment: The authors would like to thank the anonymous referee for comments which improved the presentation of this work.

\section{Proof of Theorems 1 and 2}

We begin with a general abstract result. It can be used to derive properties of logarithmic convexity of certain $L^{2}$-norms of solutions of different evolutions.

Theorem 3. Let $\mathcal{S}$ be a symmetric operator, $\mathcal{A}$ be an anti-symmetric one, both allowed to depend on the time variable, and $f(x, t)$ be a suitable function. If

$$
H(t)=\langle f, f\rangle=\|f(t)\|_{2}^{2}, \quad D(t)=\langle\mathcal{S} f, f\rangle, \partial_{t} \mathcal{S}=\mathcal{S}_{t}, \quad \text { and } N(t)=\frac{D(t)}{H(t)},
$$

then

$$
\begin{aligned}
\dot{N}(t) & =\left\langle\mathcal{S}_{t} f+[\mathcal{S}, \mathcal{A}] f, f\right\rangle / H \\
& +\frac{1}{2}\left[\left\|\partial_{t} f-\mathcal{A} f+\mathcal{S} f\right\|_{2}^{2}\|f\|_{2}^{2}-\left(\Re\left\langle\partial_{t} f-\mathcal{A} f+\mathcal{S} f, f\right\rangle\right)^{2}\right] / H^{2} \\
& +\frac{1}{2}\left[\left(\Re\left\langle\partial_{t} f-A f-\mathcal{S} f, f\right\rangle\right)^{2}-\left\|\partial_{t} f-\mathcal{A} f-\mathcal{S} f\right\|_{2}^{2}\|f\|_{2}^{2}\right] / H^{2},
\end{aligned}
$$

and

$$
\dot{N}(t) \geq\left\langle\mathcal{S}_{t} f+[\mathcal{S} ; \mathcal{A}] f, f\right\rangle / H-\frac{1}{2}\left\|\partial_{t} f-\mathcal{A} f-\mathcal{S} f\right\|_{2}^{2}\|f\|_{2}^{2} / H^{2} .
$$

Proof. We have the following identities

$$
\begin{gathered}
\dot{H}(t)=2 \Re\left\langle\partial_{t} f, f\right\rangle=\Re\left\langle\partial_{t} f+\mathcal{S} f, f\right\rangle+\Re\left\langle\partial_{t} f-\mathcal{S} f, f\right\rangle, \\
D(t)=\Re\langle\mathcal{S} f, f\rangle=\frac{1}{2} \Re\left\langle\partial_{t} f+\mathcal{S} f, f\right\rangle-\frac{1}{2} \Re\left\langle\partial_{t} f-\mathcal{S} f, f\right\rangle .
\end{gathered}
$$

Thus, multiplying (2.2) and (2.3) it follows that

$$
\dot{H}(t) D(t)=\frac{1}{2}\left(\Re\left\langle\partial_{t} f+\mathcal{S} f, f\right\rangle\right)^{2}-\frac{1}{2}\left(\Re\left\langle\partial_{t} f-\mathcal{S} f, f\right\rangle\right)^{2} .
$$

Adding an antisymmetric operator does not change the real part, and so

$$
\dot{H}(t) D(t)=\frac{1}{2}\left(\Re\left\langle\partial_{t} f-\mathcal{A} f+\mathcal{S} f, f\right\rangle\right)^{2}-\frac{1}{2}\left(\Re\left\langle\partial_{t} f-\mathcal{A} f-\mathcal{S} f, f\right\rangle\right)^{2} .
$$

Differentiating $D(t)$

$$
\begin{aligned}
\dot{D}(t) & =\left\langle\mathcal{S}_{t} f, f\right\rangle+\left\langle\mathcal{S} \partial_{t} f, f\right\rangle+\left\langle\mathcal{S} f, \partial_{t} f\right\rangle \\
& =\left\langle\mathcal{S}_{t} f, f\right\rangle+2 \Re\left\langle\partial_{t} f, \mathcal{S} f\right\rangle \\
& =\left\langle\mathcal{S}_{t} f+[\mathcal{S}, \mathcal{A}] f, f\right\rangle+2 \Re\left\langle\partial_{t} f-\mathcal{A} f, \mathcal{S} f\right\rangle,
\end{aligned}
$$


combined with the polarization identity give

$$
\dot{D}(t)=\left\langle\mathcal{S}_{t} f+[\mathcal{S}, \mathcal{A}] f, f\right\rangle+\frac{1}{2}\left\|\partial_{t} f-\mathcal{A} f+\mathcal{S} f\right\|_{2}^{2}-\frac{1}{2}\left\|\partial_{t} f-\mathcal{A} f-\mathcal{S} f\right\|_{2}^{2} .
$$

The identity (2.1) follows from (2.4) and (2.5).

Note for future use that

$$
\dot{H}(t)=2 \Re\left\langle\partial_{t} f-\mathcal{S} f-\mathcal{A} f, f\right\rangle+2 D(t),
$$

so if $\partial_{t} f-\mathcal{S} f-\mathcal{A} f=0$, one also has that $\dot{H}(t)=2 D(t)$.

Remark 1. The abstract identity in Theorem 3 shows in disguise the "frequency function" or "monotonicity argument" linked to the Carleman inequality

$$
\left\|\partial_{t} f-\mathcal{A} f\right\|_{2}^{2}+\|\mathcal{S} f\|_{2}^{2} \leq\left\|\partial_{t} f-\mathcal{S} f-\mathcal{A} f\right\|_{2}^{2} .
$$

The antisymmetric and symmetric parts of $\partial_{t}-\mathcal{S}-\mathcal{A}$, as a space-time operator, are respectively $\partial_{t}-\mathcal{A}$ and $-\mathcal{S}$. Its commutator, $\left[-\mathcal{S}, \partial_{t}-\mathcal{A}\right]$, is $\mathcal{S}_{t}+[\mathcal{S}, \mathcal{A}]$. Thus,

$$
\begin{aligned}
\left\|\partial_{t} f-\mathcal{S} f-\mathcal{A} f\right\|_{2}^{2} & =\left\|\partial_{t} f-\mathcal{A} f\right\|_{2}^{2}+\|\mathcal{S} f\|^{2}-2 \operatorname{Re} \iint \mathcal{S} f \overline{\partial_{t} f-\mathcal{A} f} d x d t \\
& =\left\|\partial_{t} f-\mathcal{A} f\right\|_{2}^{2}+\|\mathcal{S} f\|_{2}^{2}+\iint\left[-\mathcal{S}, \partial_{t}-\mathcal{A}\right] f \bar{f} d x d t \\
& =\left\|\partial_{t} f-\mathcal{A} f\right\|_{2}^{2}+\|\mathcal{S} f\|_{2}^{2}+\iint\left(\mathcal{S}_{t} f+[\mathcal{S}, \mathcal{A}] f\right) \bar{f} d x d t,
\end{aligned}
$$

and the Carleman inequality holds, when $\mathcal{S}_{t}+[\mathcal{S}, \mathcal{A}]$ is non-negative. Theorem 3 shows that $H(t)$ is logarithmically convex, when $\mathcal{S}_{t}+[\mathcal{S}, \mathcal{A}]$ is non-negative, $\partial_{t} f-\mathcal{S} f-\mathcal{A} f=$ 0 , and provided that the calculations and integrations by parts carried out in the application of Theorem 3 to a particular case can be justified.

We apply Theorem 3 in our proof of Theorem 2, and in order to justify the finiteness of the quantities involved, integrations by parts or calculations involved in this application of Theorem 3, we use Lemma 1 [10, pp.130] (and its higher dimensional version whose proof is a direct extension of the one dimensional argument given there) :

Lemma 1. Let $f$ be an entire function such that

$$
|f(x+i y)| \leq N e^{-a|x|^{2}+b|y|^{2}} \text {, with } N, a, b>0, \forall x, y \in \mathbb{R}^{n} .
$$

Then, $\widehat{f}$ is an entire function and

$$
|\widehat{f}(\xi+i \eta)| \leq N^{\prime} e^{-a^{\prime}|\xi|^{2}+b^{\prime}|\eta|^{2}}, \quad \forall \xi, \eta \in \mathbb{R}^{n}
$$

for some positive constants $N^{\prime}, a^{\prime}$ and $b^{\prime}$.

Proof of Theorem 2. We apply Theorem 3 with

$$
f(x, t)=e^{\frac{\lambda \cdot x}{\alpha t+\beta}} u(x, t), \quad \lambda \in \mathbb{R}^{n} .
$$

When the initial data to the free Schrödinger equation verifies that the right hand side of (1.10) is finite, $u_{0}$ is in $H^{\infty}\left(\mathbb{R}^{n}\right)$ and $u$ is in $C^{\infty}\left(\mathbb{R}: H^{\infty}\left(\mathbb{R}^{n}\right)\right)$. Also, $u_{0}$ extends to the complex-space $\mathbb{C}^{n}$ as an analytic function, and there are positive constants $N$, $a$ and $b$, such that

$$
\left|u_{0}(\xi+i \eta)\right| \leq N e^{-a|\xi|^{2}+b|\eta|^{2}} \quad \text { for all } \xi, \eta \in \mathbb{R}^{n} .
$$


When $T$ is positive, $f(z)=e^{\frac{i z^{2}}{4 T}} u_{0}(z)$, verifies the conditions in Lemma 1 and $u(T)$ is essentially the Fourier transform of $e^{\frac{i|y|^{2}}{4 T}} u_{0}(y)$. Thus,

$$
\left\|e^{a^{\prime}|x|^{2}} u(T)\right\|_{2}
$$

is finite for some positive number $a^{\prime}$. Corollary 2.2 in [4] shows that,

$$
\sup _{0 \leq t \leq T}\left\|e^{a^{\prime \prime}|x|^{2}} u(t)\right\|_{2}<+\infty
$$

for some new $a^{\prime \prime}$, which might depend on $a$ and $b$. By Corollary 2.2 in [4] the same occurs, when the right hand side of (1.9) is finite. Moreover, the same holds, in both cases, for all the derivatives of $u$.

Once this has been settled, our choice of $f$ in (2.7) shows that

$$
\partial_{t} f=\mathcal{S} f+\mathcal{A} f
$$

where

$$
\begin{gathered}
\mathcal{S}=-\frac{2 i}{\alpha t+\beta} \lambda \cdot \nabla-\frac{\alpha}{(\alpha t+\beta)^{2}} \lambda \cdot x, \quad \mathcal{A}=i\left(\Delta+\frac{|\lambda|^{2}}{(\alpha t+\beta)^{2}}\right), \\
\mathcal{S}_{t}+[\mathcal{S}, \mathcal{A}]=-\frac{2 \alpha}{\alpha t+\beta} \mathcal{S}
\end{gathered}
$$

and if

$$
H(t)=\int_{\mathbb{R}^{n}}|f(x, t)|^{2} d x=\int_{\mathbb{R}^{n}} e^{\frac{2 \lambda \cdot x}{\alpha t+\beta}}|u(x, t)|^{2} d x,
$$

the last comment in the poof of Theorem 3 shows that

$$
\partial_{t} \log H(t)=2 N(t)=2 \frac{\langle S f, f\rangle}{H}
$$

and

$$
\begin{aligned}
\partial_{t}^{2} \log H(t) & =2 \dot{N}(t) \geq 2\left\langle S_{t} f+[S ; A] f, f\right\rangle / H \\
& \geq-\frac{4 \alpha}{\alpha t+\beta} \frac{\langle S f, f\rangle}{H}=-\frac{2 \alpha}{\alpha t+\beta} \partial_{t} \log H(t) .
\end{aligned}
$$

In particular, (2.8) implies that the function

$$
G(t)=H(t)^{\alpha t+\beta}, \quad 0 \leq t \leq T,
$$

is logarithmically convex. Thus,

$$
G(t) \leq G(0)^{(T-t) / T} G(T)^{t / T}, \quad 0 \leq t \leq T,
$$

and consequently

$$
H(t) \leq H(0)^{\beta(T-t) / T(\alpha t+\beta)} H(T)^{t(\alpha T+\beta) / T(\alpha t+\beta)},
$$

which yields (1.7).

To prove (1.8) we recall the formulae

$$
\begin{aligned}
u(x, t) & =(2 \pi)^{-n / 2} \int_{\mathbb{R}^{n}} e^{i x \cdot \xi-i t|\xi|^{2}} \widehat{u}_{0}(\xi) d \xi \\
& =(4 \pi i t)^{-n / 2} \int_{\mathbb{R}^{n}} e^{\frac{i|x-y|^{2}}{4 t}} u_{0}(y) d y .
\end{aligned}
$$


Thus

$$
\begin{aligned}
u\left(\frac{x}{t}, \frac{1}{t}\right) & =(2 \pi)^{-n / 2} \int_{\mathbb{R}^{n}} e^{\frac{i x \cdot \xi}{t}-\frac{i|\xi|^{2}}{t}} \widehat{u}_{0}(\xi) d \xi \\
& =(2 \pi)^{-n / 2} \int_{\mathbb{R}^{n}} e^{\frac{i|x-2 \xi|^{2}}{4 t}+\frac{i|x|^{2}}{4 t}} \widehat{u}_{0}(\xi) d \xi,
\end{aligned}
$$

and so

$$
(-i t)^{-n / 2} e^{-\frac{i|x|^{2}}{4 t}} u\left(\frac{x}{t}, \frac{1}{t}\right)=(4 \pi i t)^{-n / 2} \int_{\mathbb{R}^{n}} e^{\frac{-i|x-\xi|^{2}}{4 t}} 2^{-n / 2} \widehat{u}_{0}(\xi / 2) d \xi .
$$

Hence, using the $\psi$-conformal or Appel transformation we define

$$
\bar{v}(x, t)=(-i t)^{-n / 2} e^{-\frac{i|x|^{2}}{4 t}} u\left(\frac{x}{t}, \frac{1}{t}\right),
$$

and see from (2.10)-(2.11) that $v(x, t)$ is the solution of the initial value problem

$$
\left\{\begin{array}{l}
\partial_{t} v=i \Delta v, \quad t>0, \quad x \in \mathbb{R}^{n}, \\
v(x, 0)=2^{-n / 2}{\widehat{u_{0}}}(x / 2) .
\end{array}\right.
$$

Assume now that $0<t<1$. From (1.7), with $T=1$, it follows that

$$
\left\|e^{\frac{\lambda \cdot x}{\alpha t+\beta}} u(t)\right\|_{2} \leq\left\|e^{\frac{\lambda \cdot x}{\beta}} u(0)\right\|_{2}^{\frac{\beta(1-t)}{\alpha t+\beta}}\left\|e^{\frac{\lambda \cdot x}{\alpha+\beta}} u(1)\right\|_{2}^{\frac{(\alpha+\beta) t}{\alpha t+\beta}} .
$$

Interchanging the role of $\alpha$ and $\beta$ in (1.7) and applying it with $T=1 / t>1$ to $v(x, t)$, the solution of $(2.13)$, one gets

$$
\left\|e^{\frac{\lambda \cdot x}{\beta+\alpha}} v(1)\right\|_{2} \leq\left\|e^{\frac{\lambda \cdot x}{\alpha}} v(0)\right\|_{2}^{\frac{\alpha(1-t)}{\alpha+\beta}}\left\|e^{\frac{\lambda \cdot x}{\alpha+\beta t}} v(1 / t)\right\|_{2}^{\frac{(\beta+\alpha t)}{\alpha+\beta}} .
$$

Since from (2.12)

$$
\left\|e^{\frac{\lambda \cdot x}{\beta+\alpha}} v(1)\right\|_{2}=\left\|e^{\frac{\lambda \cdot x}{\beta+\alpha}} u(1)\right\|_{2},
$$

and

$$
\left\|e^{\frac{\lambda \cdot x}{\beta / t+\alpha}} v(1 / t)\right\|_{2}=\left\|e^{\frac{\lambda \cdot x}{\alpha t+\beta}} u(t)\right\|_{2},
$$

combining (2.15)-(2.16) and the value of $v$ at the initial time, we get

$$
\left\|e^{\frac{\lambda \cdot x}{\alpha+\beta}} u(1)\right\|_{2} \leq\left\|e^{\frac{2 \lambda \cdot \xi}{\alpha}} \widehat{u}(0)\right\|_{2}^{\frac{\alpha(1-t)}{\alpha+\beta}}\left\|e^{\frac{\lambda \cdot x}{\alpha t+\beta}} u(t)\right\|_{2}^{\frac{\alpha t+\beta}{\alpha+\beta}} .
$$

From (2.14), and (2.17), and the fact that $\left\|e^{\frac{\lambda \cdot x}{\alpha t+\beta}} u(t)\right\|_{2}$ is finite (see the first paragraph at the beginning of the proof of Theorem 2), we get

$$
\left\|e^{\frac{\lambda \cdot x}{\alpha t+\beta}} u(t)\right\|_{2} \leq\left\|e^{\frac{\lambda \cdot x}{\beta}} u(0)\right\|_{2}^{\frac{\beta}{\alpha t+\beta}}\left\|e^{\frac{2 \lambda \cdot \xi}{\alpha}} \widehat{u}(0)\right\|_{2}^{\frac{\alpha t}{\alpha t+\beta}},
$$

which gives (1.8).

To prove (1.10) we square both sides of (2.18), multiply them by $e^{-|\lambda|^{2} / 2}$, integrate with respect to $\lambda \in \mathbb{R}^{n}$, and use the identity

$$
\int_{\mathbb{R}^{n}} e^{\frac{2 \lambda \cdot x}{\gamma}-\frac{|\lambda|^{2}}{2}} d \lambda=(2 \pi)^{n / 2} e^{\frac{2|x|^{2}}{\gamma^{2}}}, \quad \gamma \in \mathbb{R},
$$


to obtain in the left hand side

$$
\begin{aligned}
& \int_{\mathbb{R}^{n}} e^{-\frac{|\lambda|^{2}}{2}}\left\|e^{\frac{\lambda \cdot x}{\alpha t+\beta}} u(t)\right\|_{2}^{2} d \lambda=\int_{\mathbb{R}^{n}} \int_{\mathbb{R}^{n}} e^{-\frac{|\lambda|^{2}}{2}} e^{\frac{2 \lambda \cdot x}{\alpha t+\beta}}|u(x, t)|^{2} d x d \lambda \\
& =\int_{\mathbb{R}^{n}}\left(\int_{\mathbb{R}^{n}} e^{\frac{2 \lambda \cdot x}{\alpha t+\beta}-\frac{|\lambda|^{2}}{2}} d \lambda\right)|u(x, t)|^{2} d x=(2 \pi)^{n / 2} \int_{\mathbb{R}^{n}} e^{\frac{2|x|^{2}}{(\alpha t+\beta)^{2}}}|u(x, t)|^{2} d x,
\end{aligned}
$$

and on the right hand side a combination of a similar argument to that in (2.20) with Hölder inequality, $p=\frac{\alpha t+\beta}{\beta}, p^{\prime}=\frac{\alpha t+\beta}{\alpha t}$ leads to

$$
\begin{aligned}
& \int_{\mathbb{R}^{n}} e^{-\frac{|\lambda|^{2}}{2}}\left(\int e^{\frac{2 \lambda \cdot x}{\beta}}|u(x, 0)|^{2} d x\right)^{\frac{\beta}{\alpha t+\beta}}\left(\int e^{\frac{4 \lambda \cdot \xi}{\alpha}}|\widehat{u}(\xi, 0)|^{2} d \xi\right)^{\frac{\alpha t}{\alpha t+\beta}} d \lambda \\
& \leq\left(\iint e^{-\frac{|\lambda|^{2}}{2}+\frac{2 \lambda \cdot x}{\beta}}|u(x, 0)|^{2} d x d \lambda\right)^{\frac{\beta}{\alpha t+\beta}} \\
& \cdot\left(\iint e^{-\frac{|\lambda|^{2}}{2}+\frac{4 \lambda \cdot \xi}{\alpha}}|\widehat{u}(\xi, 0)|^{2} d \xi d \lambda\right)^{\frac{\beta}{\alpha t+\beta}} \\
& =(2 \pi)^{n / 2}\left(\int_{\mathbb{R}^{n}} e^{\frac{2|x|^{2}}{\beta^{2}}}|u(x, 0)|^{2} d x\right)^{\frac{2 \beta}{\alpha t+\beta}}\left(\int_{\mathbb{R}^{n}} e^{\frac{8|\xi|^{2}}{\alpha^{2}}}|\widehat{u}(\xi, 0)|^{2} d \xi\right)^{\frac{\alpha t}{\alpha t+\beta}} .
\end{aligned}
$$

Therefore, (2.20) and (2.21) yield (1.10).

Finally, to obtain (1.9) we reapply the argument used to get (2.20)-(2.21) but starting with (1.7) instead of (1.8).

Next, we introduce some notation: for $f \in \mathbb{S}^{\prime}\left(\mathbb{R}^{n}\right), p, q \in[1, \infty]$, and $A_{1}, A_{2}>0$ we will write

$$
f=O_{p, q}\left(A_{1} ; A_{2}\right) \text { if } e^{A_{1}|\cdot|^{2}} f \in L^{p}\left(\mathbb{R}^{n}\right), e^{A_{2}|\cdot|^{2}} \widehat{f} \in L^{q}\left(\mathbb{R}^{n}\right) \text {, with } M_{p, q}(f)=A_{1} A_{2} .
$$

In the case $p=q$ we shall write $O_{p}$ instead of $O_{p, p}$.

In this context, Hardy's result mentioned in the introduction and its extension to higher dimension found in [9] tells us that if $M_{\infty, \infty}(f)>1 / 4$, then $f \equiv 0$. Also a result in [3] affirms that in the 1 -d case if $M_{p, q}(f) \geq 1 / 4$, for any $p, q \in[1, \infty]$ with at least one of them finite, then $f \equiv 0$.

Lemma 2. If $f, g, h \in \mathbb{S}^{\prime}\left(\mathbb{R}^{n}\right)$ are such that $f=O_{p, q}\left(A_{1} ; A_{2}\right), g=O_{r, q}\left(B_{1} ; B_{2}\right)$ and $h=O_{2}\left(C_{1} ; C_{2}\right)$, with $p, q, r \in[1, \infty]$ and $1 / p+1 / r=1 / l$, then

(a) $\widehat{f}=O_{q, p}\left(A_{2} ; A_{1}\right)$.

(b) $f g=O_{l, q}\left(A_{1}+B_{1} ; A_{2} B_{2} /\left(A_{2}+B_{2}\right)\right)$ and

$$
M_{l, q}(f g) \leq \frac{B_{2}}{A_{2}+B_{2}} M_{p, q}(f)+\frac{A_{2}}{A_{2}+B_{2}} M_{r, q}(g) .
$$

(c) $g * f=O_{q, l}\left(A_{1} B_{1} /\left(A_{1}+B_{1}\right) ; A_{2}+B_{2}\right)$.

(d) $e^{i t \Delta} h=O_{2}\left(C_{1} C_{2} /\left(C_{2}+4 t \sqrt{C_{1} C_{2}}+4 t^{2} C_{1}\right) ; C_{2}\right)$, when $t>0$.

(e) $e^{-i \tau|\cdot|^{2}} h=O_{2}\left(C_{2} ; C_{1} C_{2} /\left(C_{1}+4 \tau \sqrt{C_{1} C_{2}}+4 \tau^{2} C_{2}\right)\right)$, when $\tau>0$.

Proof. Part (a) is immediate. To obtain (b) we need the following calculation: for $\mu, \nu>0$

$$
\begin{aligned}
\int_{\mathbb{R}^{n}} e^{-\mu|x-y|^{2}} e^{-\nu|y|^{2}} d y & =e^{-\left(\mu-\frac{\mu^{2}}{\mu+\nu}\right)|x|^{2}} \int_{\mathbb{R}^{n}} e^{-\left|\frac{\mu x}{\sqrt{\mu+\nu}}-\sqrt{\mu+\nu} y\right|^{2}} d y \\
& =\left(\frac{\pi}{\mu+\nu}\right)^{n / 2} e^{-\frac{\mu \nu}{\mu+\nu}|x|^{2}} .
\end{aligned}
$$


Then, taking $c=A_{2} B_{2} /\left(A_{2}+B_{2}\right)$, and combining (2.22) and Hölder's inequality we get

$$
\begin{aligned}
& \left\|e^{c|x|^{2}} \widehat{f} * \widehat{g}\right\|_{q}=\left\|e^{c|x|^{2}} \int e^{A_{2}|x-y|^{2}} \widehat{f}(x-y) e^{B_{2}|y|^{2}} \widehat{g}(y) e^{-A_{2}|x-y|^{2}} e^{-B_{2}|y|^{2}} d y\right\|_{q} \\
& \leq\left\|e^{c|x|^{2}}\left(\int e^{-q^{\prime} A_{2}|x-y|^{2}} e^{-q^{\prime} B_{2}|y|^{2}} d y\right)^{1 / q^{\prime}}\right\|_{\infty} \\
& \quad\left\|\left(\int e^{q A_{2}|x-y|^{2}}|\widehat{f}(x-y)|^{q} e^{q B_{2}|y|^{2}}|\widehat{g}(y)|^{q} d y\right)^{1 / q}\right\|_{q} \\
& \leq c\left\|e^{A_{2}|x|^{2}} \widehat{f}\right\|_{q}\left\|e^{B_{2}|x|^{2}} \widehat{g}\right\|_{q}
\end{aligned}
$$

which proves (b).

A combination of (a) and (b) yields (c). Part (d) follows from Theorem 2 estimate (1.10). Finally, (e) follows by combining (a), (d) and the formula

$$
\left.e^{-i \tau|x|^{2}} h(x)=e^{\widehat{i \tau \Delta}\left(h^{\vee}\right.}\right)(x) .
$$

Remark 2. Parts (d) and (e) in Lemma 2 still hold with $t, \tau \in \mathbb{R}$ resp. by replacing $t$ and $\tau$ on their right hand side by $|t|$ and $|\tau|$ resp.

Remark 3. The Lemma suggests to consider the class of functions verifying (2.6) as a space of test functions for more general distributions than the tempered ones. This issue will be studied elsewhere.

Corollary 1. Let $u_{0} \in \mathbb{S}^{\prime}\left(\mathbb{R}^{n}\right)$. If

$$
e^{i t_{j} \Delta} u_{0} \in L^{2}\left(e^{2 \mu_{j}|x|^{2}} d x\right), j=1,2, \quad t_{1} \neq t_{2}, \quad \mu_{1}, \mu_{2}>0,
$$

then

$$
u_{0} \in O_{2}\left(A_{1} ; A_{2}\right), \text { for some } A_{1}, A_{2}>0 .
$$

Proof. Using part (d) in Lemma 2 and Remark 2 we can assume $t_{1}=0, t_{2}=s>0$. Thus,

$$
f=u_{0} \in L^{2}\left(e^{2 \mu_{1}|x|^{2}} d x\right), \text { and } e^{i s \Delta} f \in L^{2}\left(e^{2 \mu_{2}|x|^{2}} d x\right)
$$

But

$$
\begin{aligned}
e^{i s \Delta} f(x) & =(4 \pi i s)^{-n / 2} \int_{\mathbb{R}^{n}} e^{\frac{i|x-y|^{2}}{4 s}} f(y) d y \\
& =(4 \pi i s)^{-n / 2} e^{\frac{i|x|^{2}}{4 s}} \int_{\mathbb{R}^{n}} e^{\frac{-i x \cdot y}{2 s}} e^{\frac{i|y|^{2}}{4 s}} f(y) d y .
\end{aligned}
$$

Therefore,

$$
e^{\frac{-i|x|^{2}}{4 s}} e^{i s \Delta} f(x)=(4 \pi i s)^{-n / 2} \widehat{e^{\frac{\left.i|\cdot|\right|^{2}}{4 s}}} f(x / s) .
$$

From (2.23)-(2.24) it follows that

$$
e^{\frac{i|\cdot|^{2}}{4 s}} f \in L^{2}\left(e^{2 \mu_{1}|x|^{2}} d x\right) \text {, and } \widehat{e^{\frac{i|\cdot|^{2}}{4 s}}} f \in L^{2}\left(e^{2 \mu_{2}(2 s)^{2}|\xi|^{2}} d \xi\right) \text {. }
$$


In particular, $e^{\frac{i|\cdot|^{2}}{4 s}} f=O_{2}\left(\mu_{1} ; 4 s^{2} \mu_{2}\right)$, and from Lemma 2, part (e) and the Remark 2 , it follows that

$$
u_{0}=f=O_{2}\left(4 s^{2} \mu_{2} ; \frac{4 s^{2} \mu_{1} \mu_{2}}{\mu_{1}+2 \sqrt{\mu_{1} \mu_{2}}+\mu_{2}}\right),
$$

which yields the desired result.

Proof of Theorem 1. The part (i) implies (ii) is Corollary 1. That (ii) implies (iii) is in Theorem 2, (1.10). (iii) implies $(i)$ is immediate. Lemma 2 part $(e)$ shows that $(i)$ and $(i v)$ are equivalent. Lemma 1 affirms that $(v)$ and $(v i)$ are equivalent and that each one implies $(i i)$. The fact that $(i)$ implies $(v i)$ follows from the following result (see [10], page 130):

Suppose that $f(x+i y)$ defined in $\mathbb{C}^{n}$ is an entire function such that $f(z)=O\left(e^{c_{1}|z|^{2}}\right)$ for some $c_{1}>0$ with $f(x)=O\left(e^{-c_{2}|x|^{2}}\right)$ for some $c_{2}>0$. Then $|f(x+i y)|=$ $O\left(e^{-a|x|^{2}+b|y|^{2}}\right)$ for some $a, b>0$.

(vii) implies (ii) is immediate using Lemma 2 part (b) . To see that (ii) implies (vii) define

$$
\widehat{h}(\xi)=e^{\delta|\xi|^{2}} \widehat{u}_{0}(\xi) \in L^{1}\left(\mathbb{R}^{n}\right) \cap L^{\infty}\left(\mathbb{R}^{n}\right), \quad \delta \in\left(0, b_{2}\right),
$$

So we just need to show that $h \in L^{2}\left(e^{\epsilon|x|^{2}} d x\right)$ for some $\epsilon>0$. Using that (ii) implies (vi) it follows that

$$
|\widehat{h}(\xi+i \eta)|=\left|e^{\delta(\xi+i \eta) \cdot(\xi+i \eta)} \widehat{u}_{0}(\xi+i \eta)\right| \leq N e^{(-a+\delta)|\xi|^{2}+b_{1}|\eta|^{2}}, \quad a, b_{1}>0 .
$$

Hence, taking $\delta<a$ and using that $(v)$ and $(v i)$ are equivalent we get that $h \in$ $L^{2}\left(e^{\epsilon|x|^{2}} d x\right)$ for some $\epsilon>0$ which completes the proof.

\section{Further results. Generalizations and Applications}

3.1. Some other convex weights. We return to Theorem 1, and its proof given in section 2. From the arguments used in (1.9)-(1.10) and (2.20)-(2.21), it is clear that similar estimates hold with different coefficients multiplying the Gaussian weight in each variable. More precisely, we have the following result:

Corollary 2. Using the same hypotheses and notation as in Theorem 2. Given $\vec{\gamma}=\left(\gamma_{1}, \ldots, \gamma_{n}\right) \in[0, \infty)^{n}$ (using summation convention over multiple indices) one has that

$$
\left\|e^{\frac{\gamma_{j} x_{j}^{2}}{(\alpha t+\beta)^{2}}} u(t)\right\|_{2} \leq\left\|e^{\frac{\gamma_{j} x_{j}^{2}}{\beta^{2}}} u(0)\right\|_{2}^{\theta(t)}\left\|e^{\frac{\gamma_{j} x_{j}^{2}}{(\alpha T+\beta)^{2}}} u(T)\right\|_{2}^{1-\theta(t)},
$$

for any $0 \leq t \leq T$, and

$$
\left\|e^{\frac{\gamma_{j} x_{j}^{2}}{(\alpha t+\beta)^{2}}} u(t)\right\|_{2} \leq\left\|e^{\frac{\gamma_{j} x_{j}^{2}}{\beta^{2}}} u(0)\right\|_{2}^{\mu(t)}\left\|e^{\frac{4 \gamma_{j} x_{j}^{2}}{\alpha^{2}}} \widehat{u}(0)\right\|_{2}^{1-\mu(t)},
$$

when $t \geq 0$.

Next, we shall extend Theorem 2 to the case where we replace the quadratic powers in the exponents in (1.9)-(1.10) with possibly different powers in each component.

Corollary 3. Using the same hypotheses and notation as in Theorem 2. Given $\vec{p}=\left(p_{1}, . ., p_{n}\right) \in(1,2]^{n}$ and $\vec{\gamma}=\left(\gamma_{1}, \ldots, \gamma_{n}\right) \in[0, \infty)^{n}$ there exists $c=c(\vec{p})>0$ such that

$$
\left\|e^{\gamma_{j}\left|\frac{x_{j}}{\alpha t+\beta}\right|^{p_{j}}} u(t)\right\|_{2} \leq c\left\|e^{\gamma_{j}\left|\frac{x_{j}}{\beta}\right|^{p_{j}}} u(0)\right\|_{2}^{\theta(t)}\left\|e^{\gamma_{j}\left|\frac{x_{j}}{\alpha T+\beta}\right|^{p_{j}}} u(T)\right\|_{2}^{1-\theta(t)},
$$


for $0 \leq t \leq T$, and

$$
\left\|e^{\gamma_{j}\left|\frac{x_{j}}{\alpha t+\beta}\right|^{p_{j}}} u(t)\right\|_{2} \leq c\left\|e^{\gamma_{j}\left|\frac{x_{j}}{\beta}\right|^{p_{j}}} u(0)\right\|_{2}^{\mu(t)}\left\|e^{\gamma_{j}\left|\frac{2 \xi_{j}}{\alpha}\right|^{p_{j}}} \widehat{u}(0)\right\|_{2}^{1-\mu(t)},
$$

for $t \geq 0$.

Proof. First, we notice that instead of the identity (2.19) one has the following asymptotic formula (see [10] Proposition 2, pp. 323): in the one dimensional case

$$
\int_{\mathbb{R}} e^{\lambda x-\frac{\mid \lambda p^{p^{\prime}}}{p^{\prime}}}|\lambda|^{\frac{p^{\prime}-2}{2}} d \lambda=e^{\frac{|x|^{p}}{p}}\left((2 \pi) \sqrt{p-1}+\mathrm{O}\left(|x|^{-\frac{p}{2}}\right)\right),
$$

when $1<p<\infty,|x| \geq 1$ and $1 / p+1 / p^{\prime}=1$. In particular, there is $c=c(p)$ such that

$$
c^{-1} e^{\frac{|x|^{p}}{p}} \leq \int_{\mathbb{R}} e^{\lambda x-\frac{|\lambda|^{p^{\prime}}}{p^{\prime}}}|\lambda|^{\frac{p^{\prime}-2}{2}} d \lambda \leq c e^{\frac{|x|^{p}}{p}},
$$

when $x$ is in $\mathbb{R}$. Thus, to obtain (3.1)-(3.2), one just follows the argument provided in the proof of Theorem 2 to obtain (1.9) and (1.10) respectively, but using instead of the identity (2.19), the inequality (3.3), when $n=1$ and (3.4), when $n \geq 2$ :

$$
c^{-1} e^{\frac{|x|^{p}}{p}} \leq \int_{\mathbb{R}^{n}} e^{\lambda \cdot x-\frac{|\lambda|^{p^{\prime}}}{p^{\prime}}}|\lambda|^{\frac{n\left(p^{\prime}-2\right)}{2}} d \lambda \leq c e^{\frac{\mid x p^{p}}{p}},
$$

when $1<p<\infty$ and $|x| \geq 1$.

Corollary 4. With the same hypotheses and notation as in Theorem 2. Given any $p \in(1,2]$, there is $c=c(p)>0$, such that

$$
\left\|e^{\left|\frac{x}{\alpha t+\beta}\right|^{p}} u(t)\right\|_{2} \leq c|| e^{\left|\frac{x}{\beta}\right|^{p}} u(0)\left\|_{2}^{\theta(t)}\right\| e^{\left|\frac{x}{\alpha T+\beta}\right|^{p}} u(T) \|_{2}^{1-\theta(t)},
$$

for $0 \leq t \leq T$, and

$$
\left\|e^{\left|\frac{x}{\alpha t+\beta}\right|^{p}} u(t)\right\|_{2} \leq c\left\|e^{\left|\frac{x}{\beta}\right|^{p}} u(0)\right\|_{2}^{\mu(t)}\left\|e^{\left|\frac{2 \xi}{\alpha}\right|^{p}} \widehat{u}(0)\right\|_{2}^{1-\mu(t)},
$$

for $t \geq 0$.

Note that we have stated these results for $1<p \leq 2$ since Hardy's uncertainty principle shows that for $p>2$ all the functions are 0 .

3.2. Products of solutions. Next, we shall apply in Corollary 5 the logarithmically convex inequalities to a pair of solutions $e^{i t \Delta} u_{0}$ and $e^{i t \Delta} v_{0}$ : starting with the inequality (1.7) or (1.8) for each of these solutions, multiplying the squares of their left hand sides and their right ones respectively, and following the argument in (2.19), (2.20) and (2.21), we obtain some estimates concerning the interaction of two solutions of the free Schrödinger equation.

Corollary 5. Under the same hypotheses and notation as in Theorem 2, the following inequalities hold

$$
\begin{aligned}
& \left\|e^{\frac{\lambda \cdot(x-y)}{\alpha t+\beta}} e^{i t \Delta} u_{0}(x) e^{i t \Delta} v_{0}(y)\right\|_{L^{2}\left(\mathbb{R}_{x, y}^{2 n}\right)} \\
& \quad \leq\left\|e^{\frac{\lambda \cdot(x-y)}{\beta}} u_{0}(x) v_{0}(y)\right\|_{L^{2}\left(\mathbb{R}_{x, y}^{2 n}\right)}^{\theta(t)}\left\|e^{\frac{\lambda \cdot(x-y)}{\alpha T+\beta}} e^{i T \Delta} u_{0}(x) e^{i T \Delta} v_{0}(y)\right\|_{L^{2}\left(\mathbb{R}_{x, y}^{2 n}\right)}^{1-\theta(t)},
\end{aligned}
$$




$$
\begin{aligned}
& \left\|e^{\frac{|x-y|^{2}}{(\alpha+\beta)^{2}}} e^{i t \Delta} u_{0}(x) e^{i t \Delta} v_{0}(y)\right\|_{L^{2}\left(\mathbb{R}_{x, y}^{2 n}\right)} \\
& \quad \leq\left\|e^{\frac{|x-y|^{2}}{\beta^{2}}} u_{0}(x) v_{0}(y)\right\|_{L^{2}\left(\mathbb{R}_{x, y}^{2 n}\right)}^{\theta(t)}\left\|e^{\frac{|x-y|^{2}}{(\alpha T+\beta)^{2}}} e^{i T \Delta} u_{0}(x) e^{i T \Delta} v_{0}(y)\right\|_{L^{2}\left(\mathbb{R}_{x, y}^{2 n}\right)}^{1-\theta(t)},
\end{aligned}
$$

for $0 \leq t \leq T$, and

$$
\begin{aligned}
& \left\|e^{\frac{\lambda \cdot(x-y)}{\alpha t+\beta}} e^{i t \Delta} u_{0}(x) e^{i t \Delta} v_{0}(y)\right\|_{L^{2}\left(\mathbb{R}_{x, y}^{2 n}\right)} \\
& \leq\left\|e^{\frac{\lambda \cdot(x-y)}{\beta}} u_{0}(x) v_{0}(y)\right\|_{L^{2}\left(\mathbb{R}_{x, y}^{2 n}\right)}^{\mu(t)}\left\|e^{\frac{2 \lambda \cdot(\xi-\eta)}{\alpha}} \widehat{u}_{0}(\xi) \widehat{v}_{0}(\eta)\right\|_{L^{2}\left(\mathbb{R}_{\xi, \eta}^{2 n}\right)}^{1-\mu(t)}, \\
& \begin{aligned}
\| e^{\frac{|x-y|^{2}}{(\alpha t+\beta)^{2}}} e^{i t \Delta} u_{0}(x) e^{i t \Delta} & v_{0}(y) \|_{L^{2}\left(\mathbb{R}_{x, y}^{2 n}\right)} \\
& \leq\left\|e^{\frac{|x-y|^{2}}{\beta^{2}}} u_{0}(x) v_{0}(y)\right\|_{L^{2}\left(\mathbb{R}_{x, y}^{2 n}\right)}^{\mu(t)}\left\|e^{\frac{4|\xi-\eta|^{2}}{\alpha^{2}}} \widehat{u}_{0}(\xi) \widehat{v}_{0}(\eta)\right\|_{L^{2}\left(\mathbb{R}_{\xi, \eta}^{2 n}\right)}^{1-\mu(t)},
\end{aligned}
\end{aligned}
$$

for $t \geq 0$.

Remark 4. The interaction inequalities (3.5)-(3.6) show that the $\alpha t+\beta$ power of their left hand sides are logarithmically convex functions in $[0, T]$.

Also observe that appropriate versions of these inequalities can be deduced for any finite set of solutions of the free Schrödinger equation.

In Corollary 6, we show that the "logarithmic convexity" behind the interaction inequalities in Corollary 5, implies interaction Morawetz inequalities for the free particles in the same spirit as the interaction inequalities in [2, Sections 10 and 11].

Corollary 6. Under the same hypotheses and notation than in Corollary 5, the function of $t$

is convex in $\mathbb{R}$.

$$
\left\||x-y| e^{i t \triangle} u_{0}(x) e^{i t \triangle} v_{0}(y)\right\|_{L^{2}\left(\mathbb{R}_{x, y}^{2 n}\right)}^{2}
$$

Proof. For $\gamma>0$, choose $\alpha=0$ and $\beta=\sqrt{\frac{2}{\gamma}}$ in (3.6). It shows that

$$
\left\|e^{\frac{\gamma}{2}|x-y|^{2}} e^{i t \triangle} u_{0}(x) e^{i t \triangle} v_{0}(y)\right\|_{L^{2}\left(\mathbb{R}_{x, y}^{2 n}\right)}^{2 n}
$$

is logarithmically convex in $[0, T]$. This and the fact that

$$
\left\|e^{i t \triangle} u_{0}(x) e^{i t \triangle} v_{0}(y)\right\|_{L^{2}\left(\mathbb{R}_{x, y}^{2 n}\right)},
$$

is constant, gives that

$$
\begin{aligned}
\int_{\mathbb{R}^{2 n}} \frac{e^{\gamma|x-y|^{2}}-1}{\gamma}\left|e^{i t \triangle} u_{0}(x) e^{i t \triangle} v_{0}(y)\right|^{2} d x d y & \\
\leq\left(1-\frac{t}{T}\right) & \int_{\mathbb{R}^{2 n}} \frac{e^{\gamma|x-y|^{2}}-1}{\gamma}\left|u_{0}(x) v_{0}(y)\right|^{2} d x d y \\
& +\frac{t}{T} \int_{\mathbb{R}^{2 n}} \frac{e^{\gamma|x-y|^{2}}-1}{\gamma}\left|e^{i T \triangle} u_{0}(x) e^{i T \triangle} v_{0}(y)\right|^{2} d x d y,
\end{aligned}
$$

when $0 \leq t \leq T$. The corollary follows from (3.7), after letting $\gamma$ tend to zero. 
Remark 5. Corollary 6 follows from (3.6) because the constant in front of the right hand side of the inequality is precisely equal to 1 .

Similar arguments show that

$$
\left\||x-y|^{\frac{\alpha}{2}} e^{i t \triangle} u_{0}(x) e^{i t \triangle} v_{0}(y)\right\|_{L^{2}\left(\mathbb{R}_{x, y}^{2 n}\right)}^{2}
$$

is convex, when $n \geq 3$ and $1 \leq \alpha \leq 2$. In [2] the authors proved a similar result, when $u_{0}=v_{0}$, also in the non-linear setting.

3.3. Galilean invariance. The following result, which is a consequence of Theorem 2 and the Galilean invariant property of the free Schrödinger group, describes the time evolution of the location of the "mass" of a Gaussian decaying solution.

Corollary 7. Using the same hypotheses and notation as in Theorem 2. For any $\nu \in \mathbb{R}^{n}$

$$
\left\|e^{\frac{|x+2 t \nu|^{2}}{(\alpha t+\beta)^{2}}} u(t)\right\|_{2} \leq\left\|e^{\frac{|x|^{2}}{\beta^{2}}} u(0)\right\|_{2}^{\theta(t)}\left\|e^{\frac{|x+2 T \nu|^{2}}{(\alpha T+\beta)^{2}}} u(T)\right\|_{2}^{1-\theta(t)},
$$

when $0 \leq t \leq T$, and

$$
\left\|e^{\frac{|x+t \nu|^{2}}{(\alpha t+\beta)^{2}}} u(t)\right\|_{2} \leq\left\|e^{\frac{|x|^{2}}{\beta^{2}}} u(0)\right\|_{2}^{\mu(t)}\left\|e^{\frac{4|\xi+\nu|^{2}}{\alpha^{2}}} \widehat{u}(0)\right\|_{2}^{1-\mu(t)},
$$

when $t \geq 0$.

Proof. We recall the Galilean invariance of the free Schrödinger group

$$
\begin{aligned}
u_{\nu}(x, t) & =e^{i t \Delta}\left(e^{i \nu \cdot} u_{0}(\cdot)\right)(x)=e^{-i|\nu|^{2} t} e^{i \nu \cdot x}\left(e^{i t \Delta} u_{0}\right)(x-2 t \nu) \\
& =e^{-i|\nu|^{2} t} e^{i \nu \cdot x} u(x-2 t \nu, t) .
\end{aligned}
$$

Thus combining (3.10), the identity

$$
\widehat{e^{i \nu \cdot x} u_{0}}(\xi)=u_{0}(\xi-\nu)
$$

and the inequalities (1.9)-(1.10) in Theorem 2, we obtain (3.8) and (3.9).

3.4. Final remarks. Next, we recall the following result established in [8], which is one of the main estimates in that paper :

Lemma 3. There exists $\epsilon>0$ such that if

$$
V: \mathbb{R}^{n} \times[0, T] \rightarrow \mathbb{C}, \quad \text { with } \quad\|V\|_{L_{t}^{1} L_{x}^{\infty}} \leq \epsilon,
$$

and $u \in C\left([0, T]: L_{x}^{2}\left(\mathbb{R}^{n}\right)\right)$ is a strong solution of the IVP

$$
\left\{\begin{array}{l}
\partial_{t} u=i(\Delta+V(x, t)) u+F(x, t) \\
u(x, 0)=u_{0}(x)
\end{array}\right.
$$

with

$$
u_{0}, u_{T} \equiv u(\cdot, T) \in L^{2}\left(e^{2 \lambda \cdot x} d x\right), F \in L^{1}\left([0, T]: L_{x}^{2}\left(e^{2 \lambda \cdot x} d x\right)\right),
$$

for some $\lambda \in \mathbb{R}^{n}$, then there exists $c$ independent of $\lambda$ such that

$$
\sup _{0 \leq t \leq T}\left\|e^{\lambda \cdot x} u(\cdot, t)\right\|_{2} \leq c\left(\left\|e^{\lambda \cdot x} u_{0}\right\|_{2}+\left\|e^{\lambda \cdot x} u_{T}\right\|_{2}+\int_{0}^{T}\left\|e^{\lambda \cdot x} F(\cdot, t)\right\|_{2} d t\right) .
$$


Notice that in the above result one assumes the existence of a reference $L^{2}$-solution $u$ of the equation (3.12) and then, under the hypotheses (3.11) and (3.13), shows that the exponential decay in the time interval $[0, T]$ is preserved. From the arguments used in (2.19)-(2.21), it follows that the inequality (3.14) holds with Gaussian weights, i.e. with $\gamma|x|^{2}, \gamma>0$ instead of $\lambda \cdot x$ in the exponent.

In a forthcoming work on Schrödinger equations with potentials, among other results, we shall extend those in Lemma 3 to a class of potentials $V$ without smallness assumptions, and give suitable density arguments to justify the manipulations, integrations by parts and calculations, which arise at the time of trying to derive, in this more general context, both the preservation and the logarithmic convexity of the $L^{2}\left(e^{2 \gamma|x|^{2}} d x\right)$-norm, $\gamma>0$, of the corresponding solutions.

\section{References}

[1] A. Bonami, B. Demange, and P. Jaming, Hermite functions and uncertainty principles for the Fourier and windowed Fourier transform, Rev. Math. Iberoamericana, 19 (2003), 23-55.

[2] J. Colliander, M. Keel, G. Staffilani, H. Takaoka, and T. Tao, Global well-posedness and scattering in the energy space for the critical nonlinear Schrödinger equation in $R^{3}$. To appear in Annals of Math.

[3] M. Cowling, and J. F. Price, Generalizations of Heisenberg's inequality, Harmonic Analysis (Cortona, 1982) Lecture Notes in Math. 992 (1983), 443-449, Springer, Berlin.

[4] L. Escauriaza, C. E. Kenig, G. Ponce, and L. Vega, On Uniqueness Properties of Solutions of Schrödinger Equations, Comm. PDE. 31 (2006), 1811-1823.

[5] N. Hayashi, K. Nakamitsu, and N. Tsutsumi, On solutions of the initial value problem for the nonlinear Schrödinger equations, J. Funct. Anal., 71 (1987), 218-245.

[6] L. Hörmander, A uniqueness theorem of Beurling for Fourier transform pairs, Ark. Mat. 29 (1991), no. 2, 237-240.

[7] A. D. Ionescu and C. E. Kenig, $L^{p}$ Carleman inequalities and uniqueness of solutions of nonlinear Schrödinger equations, Acta Math. 193 (2004), 193-239.

[8] C. E. Kenig, G. Ponce, and L. Vega, On unique continuation for nonlinear Schrödinger equations, Comm. Pure Appl. Math. 56 (2003), 1247-1262.

[9] A. Sitaram, M. Sundari, and S. Thangavelu, Uncertainty principles on certain Lie groups, Proc. Indian Acad. Sci. Math. Sci. 105 (1995), 135-151.

[10] E. M. Stein and R. Shakarchi, Complex Analysis, Princeton Lectures in Analysis, Princeton University Press, (2003).

(L. Escauriaza) Universidad del País Vasco / Euskal Herriko Unibertsitatea, Dpto. de Matemáticas, Apto. 644, 48080 Bilbao, Spain

E-mail address: mtpeszul@lg.ehu.es

(C.E. Kenig) Department of Mathematics, University of Chichgo, 5734 S. University Avenue, Chicago, Illinois 60637, USA.

E-mail address: cek@math.uchicago.edu

(G. Ponce) Department of Mathematics, South Hall, Room 6607, University of CaliforNia, Santa Barbara, CA 93106, USA.

E-mail address: ponce@math.ucsb.edu

(L. Vega) Universidad del País Vasco / Euskal Herriko Unibertsitatea, Dpto. De matemáticas, Apto. 644, 48080 Bilbao, Spain.

E-mail address: mtpvegol@lg.ehu.es 